\title{
Prevalencia y evolución de la apendicitis aguda en pacientes del Hospital Belén de Trujillo
}

\section{Prevalence and evolution of the acute appendicitis in the La Libertad in the years 2011 to 2015 in HBT}

\author{
Elizabeth Aguirre1 ${ }^{a}$, Cecilia Cabanillas2 ${ }^{a}$, Esteban Vergara3 ${ }^{a}$ \\ ${ }^{1}$ Hospital Belén de Trujillo, joeliah@hotmail.com \\ ${ }^{2}$ Clínica Peruano Americana - Trujillo, cecilia.cabanillas.|@outlook.com \\ ${ }^{3}$ Hospital Regional Docente de Trujillo, esivero@hotmail.com \\ a Maestría de la Universidad Privada Antenor Orrego
}

DOI: https://doi.org/10.33017/RevECIPeru2016.0006/

\begin{abstract}
Resumen
La apendicitis aguda es la emergencia quirúrgica más común en todo el mundo, y por eso la apendicectomía es uno de los procedimientos quirúrgicos más comunes. La incidencia máxima es entre la segunda y las terceras décadas de la vida. Aunque la mayoría de los pacientes con apendicitis aguda tiene enfermedad no complicada, la apendicitis complicada es relativamente común con una incidencia similar para todos los grupos de edad. Hemos encontrado que personas de edad avanzada cada vez más están siendo diagnosticados con apendicitis aguda, aunque ya en una etapa posterior de enfermedad. Algunos autores han sugerido que la apendicitis en el anciano es comúnmente asociada con un retraso en el diagnóstico y una alta tasa de complicaciones.

En nuestro estudio encontramos que el sexo masculino fue predominante, así mismo la mayor frecuencia se encontró en Trujillo, teniendo el 2012 el año de mayor número de casos.

El propósito del estudio fue determinar la prevalencia y la evolución de la apendicitis aguda en los pacientes del Hospital Belén de Trujillo, procedentes del departamento La Libertad, con datos tomados del archivo del Hospital Belén de Trujillo, durante el quinquenio 2011-2015, para poder tener una visión de la realidad de esta patología tan frecuente, pero en nuestra región.
\end{abstract}

Descriptores: apendicitis, prevalencia, evolución.

\section{Abstract}

Acute appendicitis is the most common surgical emergency around the world, and that appendectomy is one of the most common surgical procedures. The peak incidence is between the second and the third decade of life. Although the majority of patients with acute appendicitis has uncomplicated illness, complicated appendicitis is relatively common with an incidence similar for all age groups. We have found that more and more elderly people are being diagnosed with appendicitis, although in a later stage of disease. Some authors have suggested that the appendicitis in the elderly is commonly associated with a delay in the diagnosis and a high rate of complications. We also found that within the Department La Libertad, the patients from nearby provinces attend is with more incidence geographically, although we also found very remote places patients who often are the complicated.

The purpose of the study is intended to determine the prevalence and evolution of the acute appendicitis in the La Libertad Department, with data taken from the archive of the Hospital Belen of Trujillo, during the five-year period 2011-2015, in order to have a glimpse of the reality of this disease so prevalent, but in our region. 
Keywords: appendicitis, prevalence, evolution.

\section{Introducción}

La primera apendicectomía reportada se practicó en 1735, y la descripción histopatológica de la inflamación del apéndice y posterior evolución hacia peritonitis fue publicada por Reginald $\mathrm{H}$. Fitz en 18861. La apendicitis aguda es la emergencia quirúrgica más común en los Estados Unidos cada año hay más de 250000 casos diagnosticados de apendicitis aguda. El $7 \%$ de la población total tendrá apendicitis en alguna etapa de su vida, el pico de incidencia de la condición estará entre los 10 y 30 años de edad en ambos sexos con mayor frecuencia en el sexo masculino 8.6 y $6.7 \%$ para el sexo femenino.2,3,4. La apendicitis aguda constituye la condición inflamatoria aguda abdominal más comúnmente observada en el ámbito hospitalario5. Aproximadamente $7 \%$ de la población general sufre apendicitis en el curso de su vida, por lo que se considera que es la afección quirúrgica más frecuente entre las emergencias hospitalarias. Pudiendo presentarse en cualquier edad, su incidencia aumenta en menores de 5 años y alcanza un pico entre los 10 y 30 años, presentándose menor números de casos a partir de la cuarta década de la vida. 3,4 En los adultos jóvenes la relación hombre-mujer es de 4:1, la cual se iguala hacia la tercera década de la vida. En el Perú se realizaron trabajos sobre las características epidemiológicas de la apendicitis aguda en algunos nosocomios.6 De acuerdo a la clasificación internacional de enfermedades CIE-10 la apendicitis aguda se encuentra dentro del grupo de enfermedades apendiculares, apendicitis aguda complicada 0 apendicitis aguda con peritonitis generalizada 0 apendicitis aguda con absceso peritoneal 0 apendicitis aguda con peritonitis localizada. No complicada o apendicitis aguda sin especificar. El tratamiento definitivo para la apendicitis aguda es la remoción quirúrgica.

Estudios recientes muestran que una de cada 15 personas tendrá apendicitis aguda en algún momento de su vida. Hace 50 años 15 de cada
100,000 personas morían por apendicitis aguda; actualmente, la probabilidad de morir por apendicitis no complicada es inferior al $0.1 \% .4$ Un poco más del $60 \%$ de los casos son de sexo masculino. El promedio de edad es de 19 años y casi la mitad de los pacientes con apendicitis aguda tienen entre 10 y 30 años. La enfermedad es rara por debajo de los 3 años.

El objetivo del presente trabajo es Determinar la prevalencia y evolución de la apendicitis aguda en el Dpto. La Libertad del 2011 al 2015, HBT.

\section{Metodología}

Para efectos del trabajo a realizar se agrupó a las diferentes presentaciones de apendicitis aguda como complicada y no complicada. Siendo las no complicadas las apendicitis agudas sin especificar y las complicadas las demás, es decir, las que presentan abscesos o peritonitis.

Se realizó un análisis secundario de la base de datos, de los registros del Hospital Belén de Trujillo.

El estudio evaluó la Base de datos del HBTMINSA, considerándose toda la población atendida y registrada con este diagnóstico, procedente del Departamento de La Libertad.

Los datos fueron solicitados a la oficina de estadística del HBT. La elaboración de los datos, se realizó revisando todos los registros para verificar toda la información.

A continuación, se procedió a analizar, tamizar y clasificar de acuerdo a nuestras variables, utilizando los programas de Microsoft Office Excel 2007, SPSS versión 23 y Epidat 3.1. Se utilizó la Prueba de X2 para variables nominales

\subsection{Tablas}

Tabla 1: Tipo de apendicitis en el Hospital Belén de Trujillo.

\begin{tabular}{lrrrr}
\hline & \multicolumn{2}{c}{ Apendicitis aguda } & \\
\cline { 2 - 3 } & No complicada & Complicada & Total \\
\hline Año & & & \\
2011 & 439 & 108 & 547 \\
2012 & 518 & 101 & 619 \\
2013 & 485 & 48 & 533
\end{tabular}




\section{Sexo}

Hombre

\section{Procedencia}

Trujillo

2059

297

2356

Virú

Otuzco

Sánchez Carrión

25

Santiago de Chuco

\section{5}

Ascope

24

Julcan

10

Pataz

Gran Chimú

Pacasmayo

Huamachuco

Chepén

Bolívar

\section{Edad}

0-9

10-19

20-29

30-39

40-49

50-59

60-69

70-79

80-89

90-99

\begin{tabular}{cc} 
\\
9 & 502 \\
9 & 831 \\
9 & 423 \\
9 & 299 \\
9 & 197 \\
9 & 106 \\
9 & 419 \\
\hline
\end{tabular}

\begin{tabular}{rrr}
1 & 1 & 2 \\
502 & 60 & 562 \\
831 & 137 & 968 \\
423 & 73 & 496 \\
299 & 47 & 346 \\
197 & 40 & 237 \\
106 & 24 & 130 \\
41 & 11 & 52 \\
19 & 6 & 25 \\
3 & 0 & 3 \\
\hline
\end{tabular}

Pruebas Chi-cuadrado: (1) $p=0.0000,(2) p=0.0601$ (3) $p=0.0000$, por zonas costa y sierra (4) $p=0.0587$, con agrupación menores de 20 y de 70 años ó más

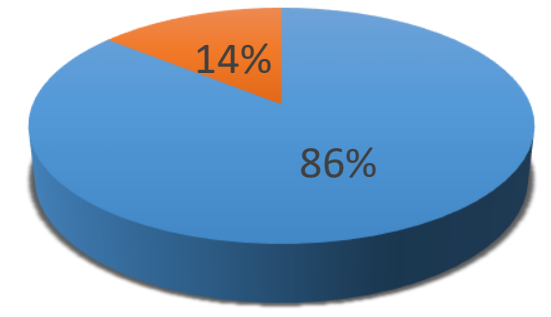

No complicada Complicada

Gráfico1: Porcentaje de Apendicitis en el Hospital Belén de Trujillo.

Tabla 2: Condición de Alta en el Hospital Belén de Trujillo.

\begin{tabular}{lll}
\hline FALLECID & MEJORADO \\
\hline
\end{tabular}




\begin{tabular}{|c|c|c|c|}
\hline 2011,00 & 2 & 363 & 182 \\
\hline 2012,00 & 2 & 473 & 144 \\
\hline 2013,00 & 1 & 417 & 116 \\
\hline 2014,00 & 0 & 460 & 100 \\
\hline 2015,00 & 1 & 501 & 60 \\
\hline \multicolumn{4}{|l|}{ SEXO } \\
\hline Masculino & 2 & 1214 & 323 \\
\hline Femenino & 4 & 1000 & 279 \\
\hline \multicolumn{4}{|l|}{ PROCEDENCIA } \\
\hline Ascope & 0 & 21 & 8 \\
\hline Bolivar & 0 & 3 & 0 \\
\hline Chepen & 0 & 2 & 2 \\
\hline Gran Chimu & 0 & 7 & 0 \\
\hline Huamachuco & 0 & 5 & 0 \\
\hline Julcan & 0 & 10 & 5 \\
\hline Otuzco & 0 & 54 & 14 \\
\hline Pacasmayo & 0 & 5 & 2 \\
\hline Pataz & 0 & 6 & 3 \\
\hline Sanchez Carrion & 1 & 40 & 3 \\
\hline SantiagodeChuco & 1 & 35 & 7 \\
\hline Trujillo & 3 & 1848 & 506 \\
\hline $\begin{array}{l}\text { Viru } \\
\text { EDAD }\end{array}$ & \multicolumn{2}{|c|}{ EDAD } & 52 \\
\hline $0-9$ & 0 & 1 & 1 \\
\hline $10-$ & 0 & 440 & 122 \\
\hline $20-29$ & 0 & 741 & 228 \\
\hline $30-39$ & 1 & 402 & 93 \\
\hline $40-49$ & 2 & 270 & 74 \\
\hline $50-59$ & 0 & 190 & 47 \\
\hline $60-69$ & 1 & 106 & 23 \\
\hline $70-79$ & 1 & 41 & 10 \\
\hline $80-89$ & 0 & 22 & 3 \\
\hline $90-99$ & 1 & 1 & 1 \\
\hline
\end{tabular}

\section{Resultados y discusión}

La apendicitis aguda constituye la patología quirúrgica más frecuente del abdomen en el Perú, y el resultado de este estudio concuerda con dicha información, pero circunscrita al Departamento de La Libertad.

En nuestro estudio, la apendicitis no complicada presento su tasa más alta en el grupo de edades de 20 a 29 años, coincidiendo con estudios previos $^{5,7}$ y la tasa más baja correspondió al grupo de edades de 0 a 9 años. La apendicitis complicada presento la tasa más alta en el mismo rango de edad, con 108 pacientes y la incidencia más baja en el rango de 90 a 99 años.

La incidencia más alta de apendicitis en el 2011 fue de 443 en la provincia de Trujillo y con la tasa más baja fue Gran Chimú y Pacasmayo con 1 solo caso.
La incidencia más alta de apendicitis en el 2012 fue de 529 en Trujillo. La provincia con la tasa más baja fue Gran Chimú, Julcan y Pataz con 1 solo caso.

La incidencia más alta de apendicitis en el 2013 fue de 454 en la provincia de Trujillo y con la tasa más baja fue Chepen y Pataz con 1 solo caso.

La incidencia más alta de apendicitis en el 2014 fue de 474 en Trujillo. La provincia con la tasa más baja fue Pataz con 1 solo caso.

La incidencia más alta de apendicitis en el 2015 fue de 457 en la provincia de Trujillo y con la tasa más baja fue Gran Chimú y Pataz con 1 solo caso. La explicación a esta mayor frecuencia en Trujillo es debido a que es la capital del departamento de la Libertad constituyéndose en localidad de referencia.

Durante los 5 años del estudio encontramos 1 fallecido al alta en las apendicitis no complicadas y 5 en las complicadas. Así mismo encontramos 
1888 mejorados en las no complicadas y 325 en las complicadas. En las no complicadas tenemos 533 curados y en la complicadas 69. Estos resultados corroboran lo esperado pues encontramos pacientes que llegan a buscar asistencia médica con cuadros muy severos y con mucho tiempo de evolución de las apendicitis, siendo así que algunos fallecen y otros requieren seguir con curaciones al alta

\section{Conclusiones}

Respecto a la apendicitis complicadas y no complicadas, la tasa más alta se encontró en el grupo de 20 a 29 años de edad en el año 2012.

La mortalidad más alta fue en las apendicitis complicadas y procedentes de lugares más alejados. La incidencia alta encontrada en cuanto a provincia le corresponde a Trujillo, debido a la ubicación geográfica del hospital de donde se obtuvo la base de datos del estudio.

En nuestro estudio encontramos que el sexo masculino fue predominante, así mismo la mayor frecuencia se encontró en Trujillo, teniendo el 2012 el año de mayor número de casos.

\section{Agradecimientos}

Al Hospital Belén de Trujillo, en su oficina de Estadistica por proporcionarnos la Base de Datos.

Al Doctor Carlos Minchon Medina por sus enseñanzas, dedicación y paciencia para motivarnos a investigar.

\section{Referencias}

[1] Ben-David, K, Sarosi GA Jr.Appendicitis.In: Feldman M, Friedman LS, Brandt LJ, eds. Sleisenger \& Fordtran's Gastrointestinal and liver disease. Vol2. 9th ed. Philadelphia, PA: Saunders Elsevier; 2010: 2599-2612.

[2] Rodríguez G, Héctor, Portillo Y. Itzel, et al, Introduction to Electrodynamics, 2nd ed. (Prentice Hall, Englewood Cliffs, NJ, 1989), Prevalencia de apendicitis aguda en un centro de segundo nivel de atención.

[3] H. Mazeh et al. Tip apppendicitis: clinical implications and maDnagement. The American Journal of Surgery. 2009;197:211215.

[4] Seetahal SA et al. Negative appendectomy: a 10-year of a nationally representative sample. The American Journal of Surgery2011;201:433-437.
[5] Rojas S. César. Epidemiología de la apendicitis aguda en el Perú 2009 - 2011. Tesis para optar el Título Profesional de Médico Cirujano, UNMSM.

[6] Farfán E. Apendicitis Aguda en el Hospital Dos De Mayo. Enero del 2000 - Julio del 2001. Tesis digitales UNMSM2002. http://sisbib.unmsm.edu.pe/bibvirtualdata/tes is/salud/Farfan_E_O/T_com pleto.pdf

[7] Gamero Marco, Barreda Jorge, HinostrozaGerardo Apendicitis aguda: incidencia y factores asociados. Hospital Nacional "Dos de Mayo" Lima, Perú 2009. Revista Horizonte Medico | Volumen 11, No 1, Enero-Junio 2011.

[8] Buckius, Michelle, McGrath, Brian, et al. Changing Epidemiology of Acute Appendicitis in the United States: Study Period 1993-2008. Journal of Surgical Research.175,185-190(2012).

[9] García-García,José;Reding-Bernal, Arturo; et al.Cálculo del tamaño de la muestra en investigación en educación médica Inv Ed Med 2013;2(8):217-224.

[10] Jasneet Singh Bhullar,Acute Appendicitis in the Elderly: Diagnosis and Management Still a Challenge THE AMERICAN SURGEON November 2014 Vol. 80, p 295-297. 